\title{
La Grèce à la Une pendant la Grande Guerre
}

Greece on the front page during World War I

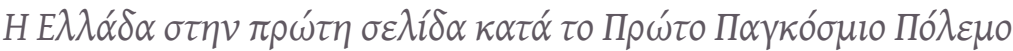

\section{Elli Lemonidou}

\section{(2) OpenEdition}

\section{Journals}

Édition électronique

URL : https://journals.openedition.org/ceb/3969

DOI : $10.4000 /$ ceb.3969

ISSN : 2261-4184

Éditeur

INALCO

Édition imprimée

Pagination : 179-198

ISBN : 978-2-85831-205-4

ISSN : 0290-7402

Référence électronique

Elli Lemonidou, «La Grèce à la Une pendant la Grande Guerre », Cahiers balkaniques [En ligne], 41 |

2013, mis en ligne le 19 mai 2013, consulté le 06 juillet 2021. URL : http://journals.openedition.org/ ceb/3969; DOI : https://doi.org/10.4000/ceb.3969

Ce document a été généré automatiquement le 6 juillet 2021

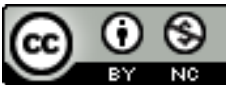

Cahiers balkaniques est mis à disposition selon les termes de la Licence Creative Commons Attribution - Pas d'Utilisation Commerciale 4.0 International. 


\title{
La Grèce à la Une pendant la Grande Guerre
}

\author{
Greece on the front page during World War I

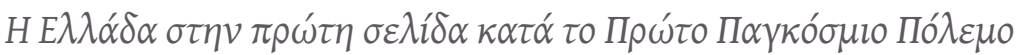

\section{Elli Lemonidou}

1 Pour commencer, nous devons indiquer que la Grèce constitue un sujet de préoccupation intense pour la France de la Première Guerre mondiale. L'étude démontre que les dirigeants français furent très concernés et particulièrement impliqués dans l'affaire grecque, et qu'il en fut de même pour la presse française, qui montra pour cette situation un intérêt et une curiosité extrêmes. Sur ce point, les propos, dans ses mémoires, de Constantinos Zavitsianos, président du Parlement grec de 1912 à octobre 1915, sont révélateurs : « Aucune des puissances étrangères n'a peutêtre occupé autant la presse française à ce moment que l'a fait la Grèce. S'il s'était agi d'une Grande Puissance ou d'une Puissance de l'attitude de laquelle aurait dépendu l'issue de la guerre, on n'aurait pas écrit plus. » (Zavitsianos, 1946, 250).

2 Quelle représentation de la Grèce les Français ont-ils pu se faire pendant la Grande Guerre, à la lumière des renseignements qui leur étaient dispensés? Quel système d'information les divers gouvernements français ont-ils mis en place concernant les affaires grecques pour orienter le regard du public français ${ }^{1}$ ?

3 Pour discerner l'image que l'on se faisait alors de la Grèce, l'étude de la presse quotidienne et périodique française, constitue évidemment la source première. Il est remarquable que l'image que l'opinion publique a pu percevoir de la Grèce et des relations de ce pays avec la France apparaisse homogène au travers de journaux de tendances politiques variées. La stratégie de cette propagande se révéla assez simple: d'une part elle consista à discréditer le roi de Grèce, présenté comme un ardent germanophile, qui n'hésitait pas à recourir à des actes anticonstitutionnels en vue de servir sa propre politique de neutralité. D'autre part, on chercha à consolider la crédibilité d'Elefthérios Venizélos, qui, lui, luttait pour le progrès et la démocratie politique dans son pays, tout en bénéficiant de l'appui de la majorité du peuple. 
4 Certes, il ne faut pas oublier que la presse n'était pas libre. À côté des organismes militaires et civils qui, dès août 1914, furent créés pour servir la propagande, il y en eut d'autres dont la fonction était la censure, avec pour objectif de maîtriser le flux d'informations nationales et étrangères et d'occulter aux Français certaines réalités de la guerre. C'est le bureau de presse, créé au sein du ministère de la Guerre, qui fut chargé de cette censure. Toute la production écrite, iconographique et orale était passée au crible de ce bureau dont le système, constamment perfectionné par les autorités françaises, se révéla très efficace (Forcade, 1998). Quant aux affaires helléniques, la censure avait pour rôle de dissimuler ce qui desservait la propagande officielle française. Elle intervint pour interdire les commentaires qui suggéraient une pression quelconque de la part de l'Entente sur la Grèce afin de l'obliger à entrer en guerre, et pour bloquer en général toute information sur la manière forte employée par les Alliés, susceptible de ternir leur image auprès de l'opinion internationale ${ }^{2}$.

Dans le présent article, nous comptons examiner de manière chronologique la production de la presse française sur les affaires helléniques, afin d'observer le contenu et la ligne idéologique de l'information fournie en France concernant la Grèce pendant la Première Guerre mondiale et de comprendre la façon dont cette information a pu influencer les masses et l'opinion publiques françaises.

\section{Octobre 1915-septembre 1916 : la presse française contre la Grèce royaliste ${ }^{3}$}

6 Durant les premiers mois de la guerre, la Grèce ne fut pas au centre de l'intérêt du public ni du gouvernement français, qui avait d'autres préoccupations beaucoup plus urgentes (la bataille de la Marne, le séjour du gouvernement à Bordeaux, les problèmes d'armement et d'alliances, etc.). De la même manière, les journaux français qui mentionnaient la Grèce, uniquement dans les quelques articles qui analysaient la question balkanique, n'annonçaient en rien ceux des années suivantes qui ont consacré régulièrement des colonnes entières de leurs premières pages à l'affaire grecque.

7 L'intérêt pour la Grèce est devenu particulièrement soutenu après le débarquement des troupes alliées à Salonique, le 5 octobre 1915, et à la suite de la deuxième démission de Venizélos, le même jour, face au refus du roi de donner son accord à la participation de son pays aux opérations alliées. Cette démission a été accueillie avec beaucoup d'émotion en France, et elle a été présentée à la Une des journaux comme « un coup d'État du roi », ou comme « un acte de révolte contre le Parlement et la nation ${ }^{4}$. C'est d'ailleurs à ce moment que l'image de la Grèce bascula : le pays apparut alors comme traître vis-à-vis non seulement de ses voisins, notamment des Serbes ${ }^{5}$, mais aussi à l'égard de la cause de l'Entente. Dans ce sens, Gustave Hervé écrivait dans son éditorial de La Guerre sociale du 8 octobre ${ }^{6}$ :

Ce n'est pas nous qui violons la nation grecque en débarquant à Salonique ; c'est le roi Constantin, beau-frère du kaiser, qui violente la nation à la tête de laquelle la France, l'Angleterre et la Russie l'ont placé, en l'empêchant de tenir la parole qu'elle a donnée aux Serbes. [...] Si nous n'étions pas des poules mouillées, et si nous aimions vraiment le peuple grec, violenté par son roi, vingt-quatre heures après le coup d'État du roi Constantin contre le Parlement de la nation grecque, les flottes alliées seraient arrivées au Pirée et les ambassadeurs de la Quadruple-Entente auraient tenu au beau-frère de l'empereur Guillaume ce discours dépouillé d'artifices : «Monsieur, si dans les vingt-quatre heures vous n'avez pas rendu la 
liberté à votre peuple, si dans vingt-quatre heures Venizélos n'a pas repris le pouvoir avec mission de tenir la parole donnée aux Serbes, nous vous renvoyons à Berlin, vous et votre gracieuse épouse ! .

8 Tout au long des mois qui suivirent, la presse française sentit qu'il était de son devoir de sensibiliser l'opinion publique, ce qu'elle fit en critiquant violemment la politique de Constantin et des ministères royalistes qui ont succédé à Venizélos et qui condamnaient, disait-elle, la Grèce à une déchéance certaine. L'avenir de l'hellénisme était désormais compromis. Une neutralité grecque trop prolongée aurait pour résultat l'établissement de l'hégémonie bulgare dans les Balkans. Les pires scénarios furent envisagés : une victoire bulgare ramènerait la Grèce aux limites qu'elle avait avant 1912 et compromettrait à jamais ses espérances européennes! De plus, la victoire turque lui ferait perdre les îles, et supprimerait les dernières chances d'affranchissement de l'hellénisme asiatique. La non-participation à la guerre serait sans doute "le suicide » de la Grèce... tout plaidait en faveur de la participation du pays à la guerre ${ }^{7}$.

Le nouveau cabinet d'Alexandre Zaïmis, qui succéda à Venizélos après sa démission a été condamné avant même d'avoir pu se défendre ${ }^{8}$. Et lorsque, le 4 novembre, à la suite d'un vote de la Chambre sur la politique générale, Zaïmis démissionna et que le roi Constantin, au lieu d'appeler Venizélos, offrit le pouvoir à Stéphanos Scouloudis, les publications devinrent encore plus virulentes à l'égard du roi et de son nouveau Premier ministre. Elles cherchèrent à convaincre leur public que la République avait désormais en Grèce des ennemis à abattre. Les déclarations de Scouloudis sur le désarmement des soldats serbes ou alliés du front d'Orient, dans l'hypothèse où ils pénétreraient sur le sol grec, n'étaient pas faites pour arranger les choses. Elles provoquèrent une exacerbation importante dans la presse et parmi les Français, puisqu'on comprit que les troupes françaises et anglaises couraient de grands risques. C'est pourquoi on recommandait la manière "forte, très forte " contre Athènes ${ }^{9}$ et on menaçait ouvertement le roi d'une vengeance des Alliés, maîtres de la mer, s'il prenait une initiative quelconque contre les Serbes: "il pourrait ne plus rester pierre sur pierre à Athènes, et si nos canons respectent l'Acropole, ils se rattraperaient sur le palais « emboché » du roi » ${ }^{10}$.

Devant cette hostilité de la presse française, Athos Romanos, ministre grec en France, eut souvent recours à son influence sur Le Temps qui bénéficiait d'un abonnement annuel de l'ambassade de Grèce et d'une subvention. En échange de cette manne, il devait adopter une attitude conciliante en faveur de la Grèce, se trouvant souvent dans la nécessité de publier des articles favorables pour ce pays, des communiqués ou des démentis envoyés d'Athènes ${ }^{11}$. Le journal recevait aussi des télégrammes gratuits de la Grèce, partageant ce privilège avec l'agence des Balkans, l'Agence d'Athènes et Le Journal des Balkans à Bucarest ${ }^{12}$.

11 Il y a cependant tout lieu de supposer que Le Temps n'était pas le seul journal subventionné par le gouvernement grec. Déjà, en 1914, une Commission de Presse avait été créée à la légation grecque de Paris, dont furent membres Romanos, Nicolaos Politis et Antoine Vlastos. Cette commission avait pour tâche de gérer l'argent mis à sa disposition par le ministère des Affaires étrangères grec pour la presse : abonnements annuels aux journaux français, dépenses pour les agences d'informations et autres. En décembre 1914, Romanos demanda la mise à disposition d'un budget de 175000 francs pour la Commission de Presse pour l'année 1915, dont une somme égale à 25000 francs environ serait consacrée pour des « actions imprévues » auprès de la presse. Le budget 
fut approuvé par Venizélos. Finalement la somme octroyée se montera à 36000 francs, payés par l'institution de crédit du nom de " crédit Chérif Pacha »" ${ }^{13}$. Lorsque Venizélos quitta le pouvoir, Scouloudis continua à envoyer 36000 francs à la Commission de presse, bien que plusieurs fois il ait demandé sa suppression ${ }^{14}$.

Néanmoins, ces subventions ne suffirent pas à supprimer les attaques contre le roi et Scouloudis. Fin mai 1916, quand les Bulgares occupèrent le fort Rupel, les journaux français retrouvèrent leur ton virulent pour parler de la Grèce. Supervisée par les officiers allemands, la retraite de la garnison grecque du fort s'était effectuée sans combats, dans les délais imposés par les Bulgares. L'incident fut décrit dans tous ses détails par la presse française, qui s'indigna de cette nouvelle "trahison " grecque. Le verdict fut vite prononcé : cette évolution était le résultat de l'entente du cabinet Scouloudis avec l'ennemi héréditaire $»^{15}$. La Grèce suivait sa destinée : la Macédoine était aux mains des Bulgares qui en avaient été honteusement chassés naguère par l'armée victorieuse de Constantin, Kavala menacée, Serrès occupée ou sur le point de l'être. Tel était le bilan de la politique suivie pendant des mois par les dirigeants grecs et le pays pouvait voir, comme Stephen Pichon l'écrivait dans le Petit Journal le 30 mai 1916, ce que lui coûtaient la foi de son roi en la puissance allemande et le refus d'écouter la voix de son grand homme d'État, Venizélos.

De plus, considérant que cette situation entraînait de graves dangers pour l'armée alliée à Salonique, les publicistes français paraissaient indignés et demandaient des garanties pour la sécurité de leurs soldats ${ }^{16}$. Même L'Eeuvre, qui avait pourtant beaucoup critiqué l'expédition à Salonique dans le passé, était maintenant d'accord: «Qu'on en finisse à Athènes» était le titre de l'article d'Henri Pozzi du 19 juin, (p.2, col.3), expliquant que "Souverain, ministres, officiers de cour, germanophiles de tout rang et de tout poil, tous ces gens qui nous haïssent et dont nous lésons les intérêts les plus chers ne s'inclineront que devant la force, et ils ne le feront sans lutte que si nous ne leur en laissons pas le temps... Finissons-en ».

Or, fin juillet 1916, les Bulgares attaquèrent la Macédoine centrale. Les troupes grecques se replièrent dans le triangle formé par Serrès, Drama et Kavala, laissant le champ libre à l'ennemi. Dans les journaux parisiens, on décrivit les scènes violentes de pillage qui eurent lieu, illustrées par des témoignages insoutenables de victimes et de réfugiés. Un mois plus tard, l'agression armée avait atteint la Macédoine orientale. Cette vision de la Grèce envahie par ses ennemis du passé et traitée comme un pays conquis était intolérable aux publicistes qui, comme le public français, s'affligèrent de cette situation et invitaient leur gouvernement à prendre des mesures décisives (Herbillon, 1930, 333).

Influencés par le ton des journaux locaux, les Français de Marseille se montrèrent hostiles envers les citoyens grecs de la ville. Les Grecs purent mesurer le changement progressif d'attitude à leur égard. Ils dépendaient de la France par leur établissement chez elle, et dépendaient de la flotte alliée par les profits qu'ils tiraient du trafic maritime. Leurs angoisses s'exprimaient dans les lettres envoyées à leurs proches : "Nous ne savons pas si le gouvernement français nous laissera demeurer tranquillement sur son territoire ou s'il nous expédiera comme c'est son droit", écrivait un Grec de Marseille, le 5 juin 1916 ${ }^{17}$. Le 27, la censure intervint et une consigne générale fut envoyée à la Commission de contrôle de presse de Marseille, en vue d'interdire dans les journaux locaux les attaques contre la communauté grecque ${ }^{18}$. Pierre de Margerie, directeur politique du Quai d'Orsay, à la demande insistante de 
Romanos, intervint également en envoyant une lettre au ministre français de la Guerre : «La censure française se trouve fréquemment obligée d'arrêter à leur arrivée des télégrammes expédiés par des correspondants de presse à Salonique en raison du ton violent, voire injurieux qu'ils affectent à l'égard de la Grèce et du roi Constantin. Quels qu'aient pu être nos griefs contre le gouvernement grec et contre le roi lui-même, nous ne pouvons laisser publier en France des commentaires de nature à indisposer contre nous-mêmes la partie de l'opinion grecque qui nous est favorable. Il y a là une question de courtoisie internationale et de tact en même temps que de convenance politique $»^{19}$.

16 Les relations diplomatiques franco-grecques ne faisant qu'empirer, il fut décidé de ne plus laisser aucune chance à la Grèce royaliste. C'est pour cette raison que le cabinet royaliste de Nicolas Calogéropoulos, qui entra en fonction le 16 septembre, connut l'hostilité et le rejet de la presse française dès ses débuts ${ }^{20}$. L'appel au pouvoir de Venizélos paraissait à l'opinion française le seul moyen de dissiper la méfiance que l'attitude de Constantin avait fait naître durant les mois précédents. On commença aussi à évoquer le retour de Vénizélos au pouvoir et l'intervention grecque aux côtés des Alliés, ainsi le Bulletin du jour dans Le Temps du 5 septembre 1916. Or, le 26 septembre, Venizélos embarquait pour la Crète, où il manifesta son intention d'aller à Salonique constituer un gouvernement provisoire.

\section{L'information en France sur la Grèce divisée}

Dès l'instant où il se produisit, la presse française, unanime, se félicita de l'éclatement du mouvement national de Salonique, présentant cet événement comme le seul capable de sauver la Grèce de la déchéance imminente à laquelle ses gouvernants l'avaient condamnée. «La nouvelle Grèce s'organise. C'est vers Salonique un afflux impétueux de toutes les forces vives que possède encore la nation » annonçait en titre l'article de l'Excelsior du 29 septembre (p. 3, col. 2, 3). «Le grand Crétois a fait le grand geste » soulignait Le Petit Journal du 29 septembre (p.1, col. 3), et il ajoutait: «Attendons l'arrivée du chef du parti libéral; peut-être son entrée en scène permettra au peuple grec de sauver du naufrage ce qui n'est pas perdu sans retour ».

18 À l'unanimité les journaux proclamèrent qu'enfin il était temps pour Venizélos d'agir : la Grèce était vouée par ses gouvernants à une déchéance certaine; la perte du fort Rupel, l'occupation de la plus grande partie de la Macédoine grecque, avaient conduit la situation à un tel point que l'existence même du pays était compromise. Venizélos ne pouvant plus supporter que cette situation s'éternise, avait accepté de répondre à l'appel du peuple. Son nouveau gouvernement n'avait qu'un seul but : ne plus laisser le Bulgare, l'ennemi héréditaire, ravager la Grèce. Il n'était aucunement dirigé contre le roi ou sa dynastie ; Venizélos l'avait déclaré avant son départ d'Athènes. C'est pourquoi, d'ailleurs, c'était à tort qu'on qualifiait le mouvement nouvellement né de « révolutionnaire » puisqu'il n'était dirigé en effet que contre les ennemis du dehors, et qu'il ne cherchait point à tirer vengeance de ceux qui étaient les auteurs du désordre qui régnait en Grèce ${ }^{21}$. Il s'agissait d'un "véritable soulèvement national » et d'un soulèvement qui ne se produisait contre aucune autorité établie, cherchant seulement à défendre la patrie menacée ${ }^{22}$.

Dans ce climat d'euphorie générale, certaines feuilles éprouvèrent pourtant le besoin d'éliminer tout soupçon concernant une implication éventuelle des Alliés dans 
l'évolution des événements en Grèce. Ce fut le cas du Figaro qui certifia que les ministres de l'Entente à Athènes assistaient à ces dramatiques événements " en simples spectateurs ", sachant bien qu'ils n'avaient pas à intervenir dans les développements de la politique intérieure de la Grèce, ou du Temps qui précisait que les Alliés « en suivent les développements avec une sympathie qu'explique leur ancienne et traditionnelle amitié pour la Grèce. Mais puisque l'Hellade se montre résolue à s'aider elle-même, ils n'ont pas à intervenir dans ce mouvement, qui reste purement national $»^{23}$.

Cependant, les premières manifestations de joie n'ont pas empêché que l'on se posât des questions: que ferait Constantin? Comment réagirait-il? Quelle attitude adopteraient les Alliés à son égard ?

D'après les journaux parisiens, le roi de Grèce devait choisir entre la rupture avec la Bulgarie ou une diminution de son autorité qui équivaudrait à la déchéance; en effet, se cantonner plus longtemps dans une neutralité malveillante vis-à-vis des Alliés, surtout après l'intervention roumaine et le succès de l'offensive du général Sarrailh, commandant de l'armée d'Orient, serait nettement servir la cause bulgare. D'ailleurs, la situation intérieure de la Grèce était devenue particulièrement précaire: le cabinet Calogéropoulos, privé de relations normales avec l'Entente, ne jouissait d'aucune influence, les ligues de réservistes formaient un État dans l'État, faisant régner l'anarchie dans le pays ${ }^{24}$; «il n'y ait jamais eu gâchis supérieur à celui de la Grèce », écrivait Pichon le 6 octobre dans l'éditorial du Petit Journal.

On attaqua encore plus sévèrement Constantin quand, après la démission de Calogéropoulos, le 4 octobre, il choisit d'appeler au pouvoir Spyridon Lambros et non pas Venizélos ${ }^{25}$. Lambros devint l'objet d'attaques violentes à cause de son âge avancé, de ses attaches avec les Empires centraux, de son métier d'historien, de la composition de son ministère qui comprenait aussi d'autres universitaires: "La formation du nouveau cabinet ressemble à un conseil de révision à rebours où l'on choisirait, non les plus vigoureux et les plus capables, mais les plus débiles, moralement et physiquement » ironisait Le Matin du 8 octobre (p. 1, col. 3). Les Alliés avaient compris que les ministères qui se succédaient les uns après les autres n'avaient pour but que de gagner du temps et d'user leur patience. À leurs yeux l'heure des décisions était arrivée, ils devaient donc prendre la situation en mains et passer à l'action, ne plus laisser les amis de l'Allemagne en Grèce nuire à ceux à qui la Grèce devait d'ailleurs son existence $^{26}$.

Ce ton violent des journaux réussit à exciter le public français, auprès duquel la Grèce jouissait alors d'une réputation nettement défavorable. Même les relations commerciales franco-grecques subirent le contrecoup de cette situation. Dès septembre 1916, les industriels en France commencèrent à refuser de nouer des relations avec les commerçants grecs, tant que leur pays n'aurait pas adopté une attitude nettement favorable à l'Entente: "Vous ne trouverez pas étonnant qu'en raison de l'attitude inamicale du souverain qui préside aux destinées de votre pays, nous n'ayons nullement l'intention de traiter des affaires avec la Grèce » écrivaient le 16 septembre les Rizeries indochinoises à un commerçant du Pirée. Quelques jours plus tard, un Français de Lyon écrivait à un marchand d'Athènes : "Nous préférons vous dire très franchement qu'il nous déplaît de faire n'importe quoi avec un gouvernement dont l'attitude actuelle est si équivoque et si contraire aux traditions de votre pays ${ }^{27}$. Le public français, de plus en plus favorable au gouvernement de Salonique, se montrait décidé à ne plus permettre à son gouvernement de ménager le roi Constantin. 
24 L'hostilité contre la Grèce royale atteignit son point culminant après les événements des $1^{\text {er }}$ et 2 décembre 1916 à Athènes $^{28}$. On qualifia l'événement de "guetapens d'Athènes ", et des titres émouvants ont été trouvés pour rendre compte de la situation : "Le sang français coule à Athènes "; on parlait de "l'agression d'Athènes " ou des "Vêpres athéniennes »" Le Temps du 8 décembre écrivait (p. 2, col.2): «Les scènes auxquelles nous assistâmes hier à Athènes, rappellent les récits historiques que nous avons lus de l'extermination des huguenots à Paris, le jour de la SaintBarthélemy ». Constantin fut désigné comme le premier responsable du massacre des marins français et les journaux, à nouveau unanimes, réclamaient à grands cris une action énergique contre ce roi "félon », et la punition exemplaire de ce "boche». Ils étaient tous l'avis qu'une leçon devait être donnée aux coupables, et clamaient avec vigueur: "Frappons seulement les coupables, mais frappons-les bien...", comme on lisait dans Le Journal des Débats du 8 décembre (p. 902). La trahison de Constantin s'aggravait encore du fait qu'il s'agissait d'un acte "prémédité » : «Que le coup ait été préparé, voulu, concerté, point de doute » écrivait Le Petit Parisien du 3 décembre (p. 1, col. 2).

25 Les journaux, à nouveau unanimes, conseillaient tous de donner une leçon aux coupables qui avaient beaucoup, et depuis longtemps, profité de l'excès de confiance et de mansuétude des Alliés ; aussi n'était-il plus question pour ces derniers de se fier à un gouvernement grec qui n'était pas absolument subordonné à leurs volontés. Ils devaient enfin se décider à prendre les affaires helléniques en mains ${ }^{30}$. Le Petit Parisien du 4 décembre 1916 (p. 1, col.4) expliquait aussi qu'ayant pris délibérément position contre l'Entente, Constantin devait s'attendre à subir les conséquences de son attitude : «Le roi des Hellènes qui nous a marqué son hostilité dans une journée qui rappelle en petit les fameuses Vêpres siciliennes ne saurait être surpris si nous lui montrons d'une manière précise notre mécontentement; c'est notre sérénité qui l'étonnerait. Il y a toujours une heure où les fortes décisions s'imposent. La voici... ». Enfin, Le Journal des Débats du 8 décembre (p.902), clamait avec vigueur: «Frappons seulement les coupables, mais frappons-les bien. Au nombre de ces coupables, et le premier de tous, est le roi Constantin. Les Alliés seraient, dit-on, résolus à donner à l'affaire une solution radicale...»

26 La nouvelle d'une mobilisation imminente grecque, répandue par les feuilles quotidiennes dès le 10 décembre, amena les journalistes français à hausser encore le ton: "Châtier Constantin! Il mobilise contre nous », tel fut le titre sans nuances de l'article de L'Écho de Paris du 10 décembre (p. 1, col. 3 et 4), et, le même jour, Le Matin (p.1, col.6) écrivait que la menace du flanc de l'armée d'Orient était devenue une réalité ; il ne fallait plus compter sur les assurances données par le roi de Grèce qui ne seraient pas plus tenues que ses promesses antérieures. Le devoir de l'Entente était désormais tout tracé "reconnaissons officiellement le gouvernement national notre ami et notre allié ; infligeons à l'autre, à celui qui est l'agent du kaiser, la sévère correction qu'exige le double souci de notre honneur et de notre sécurité » soutenait Louis Marcellin dans La Liberté du 11 décembre 1916 (p. 1, col. 3, 4).

27 Le public français, particulièrement excité sous l'effet de ces publications, ne pouvait comprendre ni accepter le fait qu'aucune réparation n'eût été obtenue pour les marins français, et que loin de recourir à la force, on continue à négocier avec Constantin ${ }^{31}$. Les lettres des Français, qui étaient interceptées par le contrôle postal de Marseille, confirment ce sentiment: «Pourquoi ergotons-nous encore devant les tombes de nos 
marins assassinés ? Comment se fait-il que le roitelet de Grèce le prenne de si haut avec nous? » demandait un correspondant de Paris, le 7 janvier, tandis qu'un autre écrivait le 13: «Que nos gouvernants prennent garde [...] l'opinion publique ne leur pardonnerait pas une nouvelle erreur de ce genre $\aleph^{32}$. La même excitation gagna les soldats français du front occidental, qui, après les événements de décembre à Athènes, traitaient le roi de Grèce de «traître à sa parole » et accusaient la Grèce de sa politique "sournoise ", la considérant désormais comme "un nouvel ennemi » (Cochet, 1986, 431-432).

Néanmoins, après la mi-janvier 1917, dans les lettres des Français civils ou militaires, et surtout dans les journaux, les passages relatifs à la question grecque commencèrent à se faire de plus en plus rares. L'intérêt porté à ce pays décroissait, l'espoir et l'inquiétude petit à petit disparurent. Plus précisément, la presse française, soumise à une censure vigoureuse, fut obligée de cesser ses attaques contre le roi de Grèce, mais aussi de s'abstenir de tout commentaire favorable au gouvernement de Venizélos. Ce changement était dû d'un côté au fait que le gouvernement d'Aristide Briand, ayant décidé d'entamer des négociations avec Constantin, jugeait prudent d'éviter les tensions, de l'autre, à l'attitude de l'Italie qui, durant la conférence de Rome, avait bien mis en évidence qu'elle ne pourrait pas supporter une grande Grèce ou un puissant Venizélos.

Au début, l'opinion publique française supporta très mal cette nouvelle position; les collaborateurs de Venizélos, depuis Paris, le rapportaient à Salonique : le public, les hommes politiques et les journalistes ne cachaient pas leur colère ${ }^{33}$. Or, très vite, ils finirent par adopter une attitude d'indifférence, parce qu'ils en vinrent à penser qu'il fallait ne défendre que les seuls intérêts français et moins s'intéresser aux intérêts de la Grèce dont le peuple grec seul avait la responsabilité. D'ailleurs, durant la période suivante, l'attention des Français fut absorbée par des questions graves, qui exigeaient une solution urgente : les problèmes de ravitaillement en France, l'offensive générale des armées alliées projetée pour le printemps 1917, la révolution russe et la perspective de l'entrée des États-Unis en guerre (Renouvin, 1970, 313-315). Face à ces questions, l'affaire grecque devenait aux yeux des Français une affaire "petite et secondaire", systématiquement écartée des discussions (Petsalis-Diomidis, 1988, 224). Et c'est seulement à la fin mars 1917 qu'à la faveur d'une série de conjonctures nouvelles, la presse française cessa de se taire pour faire sa place à une campagne pro-venizéliste.

\section{Le tournant de mars 1917 : la presse française au service du venizélisme}

30 La politique de Briand en ce qui concerne l'affaire hellénique, fut en grande partie la cause de sa chute. Ses partisans l'accusèrent d'une attitude faible et équivoque à l'égard du roi de Grèce qui aurait fait du tort à la dignité française. C'est pourquoi, après sa démission, l'opinion et la presse française demandèrent unanimement que, sur ce point, l'on changeât de conduite politique. Pour Alexandre Ribot, qui arriva au pouvoir le 20 mars 1917, l'affaire grecque acquit donc une importance primordiale. Le nouveau Président du conseil avait dessein d'adopter une tactique différente, pour obtenir des résultats différents ; pour atteindre son but, il paraissait prêt à recourir même à des mesures radicales, en vue de faire réussir ses plans et d'emporter l'approbation de l'opinion publique (Cambon, 1946, 167). 
31 Cette péripétie qui se jouait sur la scène politique française, combinée à une nouvelle conjoncture défavorable à l'absolutisme en Grèce et favorable au contraire au venizélisme - l'intervention américaine et la révolution russe, les opérations heureuses de l'Entente avec le recul des Allemands - firent que les journaux français témoignèrent d'un renouveau d'intérêt pour les affaires grecques, tout en adoptant une attitude nouvelle, dont les premiers signes furent l'accentuation de la sévérité contre Constantin et en même temps le relâchement considérable de la censure, qui permit dorénavant qu'on exprimât ses griefs contre le roi de Grèce ${ }^{34}$.

32 Ainsi, quelques jours à peine après l'investiture de Ribot, les journaux commencèrent à publier des informations sur la révolte dans les îles de Zante, Céphalonie, Skiathos ou Cérigo, contre la "tyrannie » de Constantin et l'adhésion de ces îles au mouvement « libérateur » de Salonique (Cosmin, 1969, 352). Le Temps du 30 mars dans son Bulletin du jour intitulé: "Le paradoxe grec», lança une attaque pleine d'aigreur contre Constantin, mais aussi contre la politique alliée qui lui avait permis de rester «le maître du jeu ", malgré toutes ses trahisons : "jusqu'ici, on a tout fait pour livrer la Grèce à son roi allemand. On a même maintenu de force sous son autorité ceux de ses sujets qui en étaient las. Voilà le paradoxe malheureusement renforcé par une longue politique à laquelle il serait désirable que, d'accord avec nos alliés, M. Ribot pût mettre fin. ".

33 Le 11 avril, Le Temps revenait à la charge dans son Bulletin du jour pour publier l'article d'André Tardieu «Le dossier du roi de Grèce ». D’après Pierre Métaxas, chargé d'affaires de la légation grecque à Paris ${ }^{35}$, cette publication dépassait en violence et en mauvaise foi tout ce que le journal avait écrit jusqu'alors contre le gouvernement d'Athènes ${ }^{36}$. En fait, l'article - qui avait été coupé en entier par la censure, mais l'interdiction n'avait pas été appliquée ${ }^{37}$ - soutenait que puisque la censure laissait dorénavant la liberté de s'exprimer, l'occasion paraissait bonne pour passer en revue une série de faits qui prouvaient que l'attitude du roi de Grèce et de ses ministres n'avait jamais cessé d'être hostile à l'égard de l'Entente. Ainsi, on accusa Constantin d'avoir gardé contact avec Berlin dès les débuts de la guerre grâce au télégraphe sans fil du palais royal, d'avoir été le responsable des événements de décembre 1916, et d'avoir préparé subrepticement, et avec le concours de l'attaché militaire allemand, la mobilisation grecque dans le but de faciliter une attaque brusquée sur les arrières de l'armée d'Orient.

34 Pour le roi et la cour de Grèce, cet article avait une signification particulière : il constituait le premier témoignage de la nouvelle orientation de la politique française sous Ribot, et il avait pour objectif de justifier à l'avance une intervention prochaine des Alliés en Grèce ; c'est pourquoi il provoqua une émotion extrême. Le 24 avril, Eugène Zalocostas, ministre grec des Affaires étrangères, envoya aux légations grecques en Europe et aux États-Unis une notice émanant du maréchalat de la cour royale, dans laquelle le roi démentait catégoriquement les propos qui lui avaient été attribués dans l'article du Temps ${ }^{38}$.

35 Mais le signal avait été donné et ces démarches ne purent aucunement arrêter l'élan des journalistes en France. Le 19 avril L'Écho de Paris publiait (p. 2, col.3) un article intitulé "Le crépuscule du Roi Constantin », qui se concluait ainsi : "La propagande contre la dynastie s'étend de plus en plus dans le monde hellénique et l'on voit augmenter d'une manière significative le nombre des personnes qui réclament un régime républicain ». Le lendemain, dans le même journal, l'article de Jean Herbette 
(p. 1, col. 5) portait ce titre significatif: «Faites la République, Venizélos!»; il se terminait par la phrase suivante: «Vous voulez refaire la Grèce, Venizélos? Faites la République ».

Fin avril, on commença à évoquer un départ du roi de Grèce. Le 29, tous les journaux français reproduisaient des extraits d'un article paru dans le Journal de Genève d'après lequel le roi aurait songé à abdiquer le jour de la Saint-Georges au profit du prince héritier, lequel ferait appel à Venizélos pour rétablir l'union du royaume ${ }^{39}$. Pierre Métaxas sentit alors le besoin de prévenir son gouvernement sur les dangers de cette nouvelle situation ${ }^{40}$ :

Je considérerais comme une omission du devoir si en ce moment je passais sous silence le fait que la méfiance et la désapprobation contre la Grèce officielle sont arrivées en France à un point et à une limite dangereux et que par conséquent la situation où en sont présentement nos affaires est on ne peut plus cruciale. À partir des divers télégrammes et documents, l'ambassade du gouvernement royal a pris connaissance du langage que la presse française utilise à notre sujet et auquel la censure n'impose aucune mesure. Cette attitude de la presse, aussi partiale et injuste qu'elle puisse être, n'en forme pas moins une opinion publique qui nous est hostile, exploitée par ceux qui ont un intérêt contraire au nôtre.

À mesure que les rapports entre l'Entente et la Grèce royale se tendaient, à la vive contrariété des royalistes répondait la joie croissante des venizélistes de France. Athanassios Politis, attaché à la légation venizéliste de Paris, écrivait le 21 avril : «Au point de vue de notre cause, les affaires vont aussi bien. La Russie n'est plus notre ennemi et en France, la censure sur les affaires grecque est supprimée. La presse d'ailleurs attaque violemment le roi Constantin; et même Herbette hier dans l'Écho de Paris, a publié en première page un article très fort sur la Grèce, dans lequel il dit entre autres: «Faites la République, Venizélos!» » ${ }^{41}$. Un autre venizéliste de Marseille, témoignait du même optimisme: «La presse française et la presse anglaise ont heureusement changé de ton ces derniers temps. On attaque maintenant Constantin ouvertement et on réclame un plus grand appui des Alliés en faveur de Venizélos et il serait temps que cela se fit. Clemenceau et Le Temps sont surtout très ardents dans leur campagne anti-constantinienne et pro-venizéliste, et avec le ministère Ribot nous espérons que l'on arrivera à une solution, mais à une solution radicale. $»^{42}$. En même temps, Charalambos Simopoulos, chargé d'affaires grec à Londres, transmettait à Venizélos l'impression qu'il retirait de la lecture des principaux journaux français et britanniques: "il est permis de tirer la conclusion qu'une action immédiate, qui porterait une solution définitive à la question hellénique par le moyen de l'intervention directe des puissances, est imminente » (Petsalis Diomidis, 1988, 276).

En effet, la solution à la question hellénique fut donnée lors des deux conférences interalliées de mai 1917, - la conférence de Paris, les 4 et 5 mai, et celle de Londres, les 28 et 29 mai -, durant lesquelles les Alliés décidèrent d'envoyer Charles Jonnart en Grèce, en qualité de haut-commissaire, pour provoquer la déposition de Constantin, finalement réalisée le 12 juin. Dès que la presse française apprit la vraie raison de la mission de Jonnart en Grèce ainsi que le consentement de Constantin à l'ultimatum qui lui avait été adressé et qui entraînait sa déposition, unanime, elle se félicita de cette évolution: « l'abcès grec vient enfin d'être crevé » écrivait l'Humanité du 13 juin (p. 1, col. 3), qui ajoutait : « Il a fallu faire comprendre au beau-frère de Guillaume II que la politique d'atermoiements et de subterfuges employée jusqu'ici ne pouvait plus continuer et qu'il fallait ou jouer franc-jeu ou s'en aller ». 

l s'agissait d'une "victoire morale pour les alliés", comme Le Petit Parisien le proclamait le lendemain, 13 juin (p.1, col.2), avant de justifier cette expression: « D'abord parce qu'ils (les Alliés) dépossèdent un monarque qui avait usé vis-à-vis d'eux de duplicité et de félonie et qui avait essayé de nous poignarder dans le dos; ensuite parce que l'affaire a été supérieurement préparée par le général Sarrailh et que l'accumulation même des moyens a conjuré toute collision sanglante ; en troisième lieu, parce que le roi déchu était le beau-frère du kaiser et que son abdication constitue une grave humiliation pour ce dernier ».

D'après Marcellin dans La Liberté du 13 (p. 1, col. 6) : « Le rêve d'une Grèce germanique a pris fin ; une ère nouvelle s'ouvre, pleine de promesses. C'est une grave défaite pour nos ennemis». Le lendemain, Le Temps écrivait dans son Bulletin du jour: "La domination allemande est terminée en Grèce. L'assassinat des marins français est vengé. Notre pays se félicite de ces résultats. Il en sait gré à son gouvernement, qui a fait respecter les droits d'un peuple opprimé et l'honneur du drapeau national. On n'a pas besoin de justifier la chute de Constantin. Il a pris soin de la justifier lui-même, prenant plus de deux ans, en étalant comme des bravades toutes ses illégalités et toutes ses trahisons».

41 Il est pourtant à signaler la réaction quelque peu divergente de plusieurs journalistes, qui au lieu de célébrer sans réserve le dénouement de l'affaire grecque, se crurent obligés de justifier l'action entreprise. Ils se mirent donc à expliquer les motifs des Alliés concernant l'abdication forcée de Constantin. Ainsi, le Petit Journal consacra un long développement à ce sujet dans son édition du 1 juin (p. 1, col. 1 ) d'où nous tirons l'extrait suivant :

42 Les Grecs se rendent compte que les mobiles qui ont fait agir les puissances protectrices n'ont rien d'intéressé. Nos résolutions étaient, en effet, strictement conformes aux droits que nous tenons des traités. Elles ne constituaient aucune atteinte à la neutralité grecque, mais répondaient à nos obligations de puissances garantes de la liberté constitutionnelle dans le royaume. Elles s'inspiraient, en même temps, du souci d'assurer la sécurité de notre armée de Salonique, d'empêcher le retour de faits tels que ceux qui ont risqué de la compromettre, et de châtier les criminels qui en ont la responsabilité, si haut placés qu'ils puissent être.

ela même manière, Jacques Bainville précisait dans L'Excelsior le 13 juin 1917 (p. 2, col 1,2) qu'il n'y avait aucun abus de pouvoir dans l'opération que le haut-commissaire des trois puissances venait de mener à bien. Il n'y avait que l'exercice d'un droit « légitime et consacré ». La légalité se trouvait donc rétablie à Athènes et le devoir de la rétablir s'était imposé à la France, à l'Angleterre et à la Russie, garantes d'une Constitution que le roi Constantin n'observait plus.

On jugea également nécessaire d'évoquer plus précisément la question du «désintéressement total » de la France, qui n'espérait retirer aucun profit de cette opération. Ainsi, dans son éditorial du 14 du Petit Journal qui portait le titre «La soumission du roi ", Pichon écrivait-il que l'opération qui se poursuivait en Grèce n'était pas engagée dans l'intérêt spécial de la France, elle l'était dans l'intérêt général et évident de tous les pays qui, suivant le mot de Wilson, étaient obligés de vaincre l'Allemagne ou de se soumettre à elle. Dans Le Temps du 13 (Bulletin du jour) les commentaires portaient sur l'« intérêt désintéressé » de la France quant aux questions balkaniques: "De toutes les puissances alliées, grandes ou petites, dont les troupes combattent sur l'une ou l'autre face des Balkans, c'est même elle (la France) qui est le 
plus complètement désintéressée dans les affaires balkaniques. Ce désintéressement, en donnant plus d'indépendance à son jugement, mérite de donner plus de portée à ses conseils. L'opinion française en a conscience ». Dans les jours qui suivirent, le journal revint constamment sur la même idée: la France ne recherchait aucun avantage particulier dans les Balkans, quoi qu'en aient pu dire les agents de la propagande allemande ${ }^{43}$.

De surcroît, hormis ce besoin d'expliquer l'intervention alliée en Grèce, il est à constater que relativement à l'importance de l'événement, le nombre d'articles qui lui furent consacrés paraît quelque peu limité. Cette attitude réservée de la presse peut s'expliquer doublement. D'abord, dans l'opération de la déposition de Constantin qui venait d'avoir lieu, l'unité et l'unanimité des Alliés s'étaient à plusieurs reprises révélées fragiles et les difficultés à s'entendre nombreuses, d'où d'ailleurs des atermoiements continuels ${ }^{44}$. Pour certains, la principale cause des tergiversations dans la politique de l'Entente avait été les hésitations du gouvernement russe, expliquées par les liens de parenté qui unissaient Constantin à la cour royale de Russie ${ }^{45}$; pour d'autres, l'attitude indécise des Alliés était surtout due à l'Italie et à la méfiance de ce pays à l'égard de Venizélos La deuxième raison qui explique les réticences des journaux, est le fait que l'événement eut lieu à la mi-juin 1917, c'est-à-dire pendant une période où la France traversait une grave crise: grèves, mutineries militaires, crise politique, dépression morale de la population ${ }^{46}$. Il paraît donc logique que le public et la presse français aient témoigné un intérêt assez tiède pour les affaires grecques, qui ne se trouvaient pas alors au centre de leurs préoccupations.

Après l'abdication de Constantin, l'intérêt de la presse et de l'opinion publique française pour la Grèce décrut fortement. Certes on consacra quelques articles au retour de Venizélos à Athènes et à sa décision de rompre avec l'Allemagne et d'entraîner l'intervention de la Grèce dans la guerre aux côtés de l'Entente ${ }^{47}$. Surtout, à partir de fin juin 1917, les articles sur la Grèce se firent particulièrement rares. Ils parurent sporadiquement dans des journaux amis de la Grèce (Lemonidou, 2007, 376-382), ne rappelant en rien les colonnes entières consacrées à ce pays tout au long des années précédentes.

\section{Conclusion}

Il ne fait aucun doute que la presse française resta de loin LE moyen qui réussit véritablement à agir sur l'esprit des Français à l'égard de l'affaire hellénique pendant la Première Guerre mondiale, et même à toucher leurs cœurs. Quand il s'agissait de la Grèce, ce pays si éloigné géographiquement, sur lequel on ne disposait comme source d'information que de la presse, il était plutôt difficile, sinon impossible, d'entrevoir les messages occultés de l'information officielle. C'est pourquoi la campagne que la presse française mena porta ses fruits très tôt et sans difficulté ; c'est du moins ce que nous percevons au travers d'une série d'exemples qui montrent le niveau élevé d'engagement des Français dans l'affaire grecque.

Par ailleurs, conscients du pouvoir de la presse, les dirigeants français ont utilisé ce moyen pour manipuler l'opinion et servir leur politique. Ainsi, en janvier 1917, Briand, ayant choisi d'adopter une attitude de modération à l'égard de Constantin, décida d'interdire dans les journaux les attaques contre la Grèce royaliste, comme tout commentaire favorable à Venizélos, ce qui provoqua la colère et des journalistes et du 
public. Et quand le gouvernement Ribot prit le pouvoir, et voulut de nouveau sensibiliser l'opinion à l'affaire grecque, il eut également recours à la presse qui montra, sous l'impulsion gouvernementale, un renouveau d'intérêt pour la Grèce, par le biais d'une campagne qui dépassait en violence tout ce que la presse avait écrit jusqu'alors contre les royalistes d'Athènes.

Cependant, de manière inverse, c'est aussi par l'opinion ainsi formée, qu'elle réussit à influencer de manière significative l'élaboration de la politique étrangère de la France et, par conséquent, les relations bilatérales franco-grecques de cette période. À cause de la dynamique de l'opinion publique, au début de l'année 1917, l'affaire grecque prit une importance primordiale sur la scène politique française. Le public, qui avait subi l'influence de la campagne extrêmement défavorable à ce pays après les événements de décembre 1916, ne pouvait accepter le fait qu'on continuât à négocier avec Constantin et accusait Briand d'adopter un comportement qui faisait tort à la dignité française. Ce mécontentement constitua d'ailleurs l'une des premières raisons qui entraînèrent l'affaiblissement $\mathrm{du}$ cabinet Briand et sa chute, en mars 1917. L'affaire grecque constitua la première question au sujet de laquelle le gouvernement de Ribot fut invité à faire ses preuves. Sous la pression de l'opinion, il se vit obligé d'adopter une tactique différente de celle de son prédécesseur et une politique drastique qui déboucha sur la phase finale de l'affaire grecque.

Il est évident ainsi que les autorités françaises dans une guerre mettant en œuvre des techniques nouvelles, telles que la propagande et la censure, avaient réussi à établir un système d'information qui, au moins dans le cas précis de la Grèce, réussit à modeler l'opinion publique du pays et à s'imposer à elle.

\section{BIBLIOGRAPHIE}

Becker, Jean-Jacques, (1997), 1917 en Europe. L'année impossible. Bruxelles : Complexe.

Becker, Jean-Jacques, (1992), « La perception, à travers la presse française, des rapports entre la France et la Grèce pendant la Grande Guerre » dans La France et la Grèce dans la Grande Guerre, actes du colloque tenu en novembre 1989 à Thessalonique. Thessalonique : Université de Thessalonique, p. 89-100.

Becker, Jean-Jacques,(1987), « L'Opinion publique française en 1917 », Historiens et Géographes, juillet-août 1987, nº 315 : « Sur l'année 1917 », p. 1497-1507.

Becker, Jean-Jacques, (1980), Les Français dans la Grande Guerre. Paris : Laffont.

Boussenot, Georges, (1938), « Le Drame du $1^{\text {er }}$ décembre 1916 à Athènes ", Revue d'Histoire de la Guerre mondiale, $\mathrm{n}^{\circ}$ 1, janvier 1938, p. 1-27.

Cambon, Paul, (1946), Correspondance (1870-1924), vol. III : Les guerres balkaniques. La Grande Guerre. L'organisation de la paix. Paris : Grasset.

Cochet, Annick, (1986), L'opinion et le moral des soldats en 1916 d'après les archives du contrôle postal, Thèse non publiée. Paris : Université Paris X. 
Cosmin, S., (1969), Dossiers secrets de la Triple Entente. Grèce. 1914-1922. Paris : Nouvelles éditions latines.

Dartige du Fournet, Louis, (1920), Souvenirs de guerre d'un amiral (1914-1916). Paris : Librairie Plon. Forcade, Olivier, (1998), La censure politique en France pendant la Grande Guerre. Thèse non publiée. Paris : Université Paris X-Nanterre.

Herbillon, colonel, (1930), Souvenirs d'un officier de liaison pendant la Guerre Mondiale, tome I : Sous le commandement du général Joffre. Paris : Tallandier.

Lemonidou, Elli, (2007), La Grèce vue de France pendant la Première Guerre mondiale; entre censure et propagandes, Thèse non publiée. Université Paris IV.

Léon (Leontaritis), Georges, (1974), Greece and the Great Powers. 1914-1917. Thessalonique: Institute for Balkan Studies.

Maccas, Léon (1917), « Les événements d'Athènes des $1^{\mathrm{er}}$ et 2 décembre 1916 », Revue des Deux Mondes, mars-avril 1917, tome XxxviII, p. 96-135.

Maccas, Léon, (1916), «L'Alliance gréco-serbe. Les faits. Les prétextes juridiques et politiques de l'abstention », Le Correspondant du 23 février 1916.

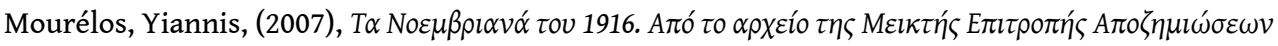
$\tau \omega \nu \theta \nu \mu \alpha \dot{\tau} \tau \omega \nu$ (Les événements du novembre 1916. Archives de la Commission Mixte d'Indemnités des victimes). Athènes : Patakis.

Mourélos, Yiannis, (1992), «Les événements de décembre 1916 à Athènes : les travaux et les jours de la Commission Mixte des Indemnités ", La France et la Grèce dans la Grande Guerre, actes du colloque tenu en novembre 1989 à Thessalonique. Thessalonique : Université de Thessalonique, p. $111-120$

Mourélos, Yiannis, (1983), L'intervention de la Grèce dans la Grande Guerre (1916-1917). Athènes : Institut français d'Athènes.

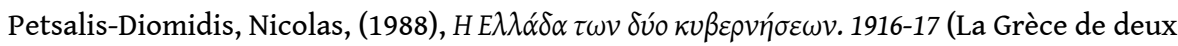
gouvernements. 1916-17). Athènes : Philipotis.

Platykas, Panayotis Dinos, (1916), La neutralité hellénique et le traité gréco-serbe de 1913. Fribourg : impr. Delaspre.

Renouvin, Pierre, (1970), «L'opinion publique en France pendant la guerre 1914- 1918 », Revue d'Histoire diplomatique, oct.-déc. 1970, $n^{\circ}$ 4, p. 289-33.

Renouvin Pierre, (1968) «L'opinion publique et la guerre en 1917 », Revue d'histoire moderne et contemporaine, janvier-mars 1968, tome xv, p. 4-23.

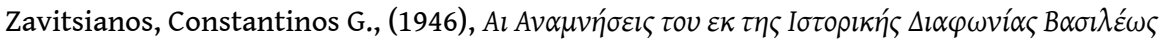

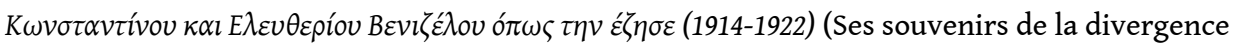
historique entre le roi Constantin et Eleuthérios Vénizélos, tel qu'il l'a vécue. 1914-1922), vol. 1. Athènes.

\section{NOTES}

1. Il serait utile de décrire en quelques mots la situation en Grèce pendant la Première Guerre mondiale. Le déclenchement de la guerre trouva les dirigeants du pays divisés : d'un côté le roi Constantin, qui prônait obstinément la neutralité, de l'autre le Premier ministre, Venizélos, qui 
n'envisageait aucune autre politique possible que l'intervention du pays aux côtés des Alliés. Le conflit entre les deux hommes s'aggravant sans cesse, deux camps antagonistes se sont constitués au sein du peuple: royalistes et venizélistes, en d'autres termes, les neutralistes et germanophiles, et les interventionnistes et francophiles. Le fossé qui les séparait se creusa, jusqu'à la rupture définitive, quand Venizélos, en octobre 1916, décida de se rendre à Salonique pour y créer un gouvernement provisoire, autrement dit un État illégal dans l'État. Dès lors le pays fut plongé dans une grave crise politique, sociale et constitutionnelle dont les conséquences devaient se faire sentir pendant plusieurs années. La situation empira à cause de l'attitude des puissances de l'Entente. Une fois engagées militairement dans la région avec l'expédition de Salonique, en octobre 1915, elles n'hésitèrent pas en effet à s'immiscer directement dans les affaires internes grecques, et à adopter une politique autoritaire. Leur but était d'obliger progressivement la Grèce à se départir de sa neutralité pour intervenir dans la guerre à leurs côtés. L'épisode de la déposition forcée du roi Constantin en juin 1917, constitua sans doute le comble de cette politique d'ingérence des Alliés.

2. Lemonidou, 2007, 124-144, 270-287, 382-387.

3. Liste des abréviations :

MAE : Archives du ministère des Affaires étrangères de France

AYE : Archives historiques du ministère des Affaires étrangères de Grèce

SHM : Service historique de la Marine nationale - France

SHD : Service historique de la Défense - France

BDIC : Bibliothèque de documentation internationale contemporaine - France

4. L'Humanité du 7 octobre 1915, p .1, col. 3.

5. Au début de la guerre la Grèce était engagée vis-à-vis de la Serbie par un traité d'alliance conclu en juin 1913, voir : Maccas, 1916 et Platykas, 1916.

6. Le journal ne s'étant pas conformé aux instructions du bureau de presse de censurer cet article, une suspension de quatre jours lui fut imposée, voir: SHD, $5 \mathrm{~N} 380$, Le ministre de la Guerre (section presse) au gouverneur militaire et au $2^{\mathrm{e}}$ bureau de l'état-major, nota s.n. Du 8 octobre 1915.

7. Le Petit Parisien du 11 octobre 1915, p. 1, col. 3.

8. « Peuples et Rois », éditorial de Fitz-Maurice dans Le Figaro du 15 octobre 1916.

9. Le Journal des Débats du 11 novembre 1915, p. 1, col. 2.

10. La Croix du 5 novembre 1915, p. 1, col. 4.

11. AYE, 1915, Г/106, 2, Romanos à Vénizélos, $n^{\circ} 4326$ du 10 décembre 1914. AYE, 1915, Г/108, 4, Romanos à Gounaris, $n^{\circ} 1262$ du 5 juin 1915 et $n^{\circ 0} 680$ du 12 juin 1915. AYE, 1915, A/6, Scouloudis à Romanos, $\mathrm{n}^{\circ} 12492$ du 27 novembre 1915.

12. AYE, 1915, $\Delta / 102$, Scouloudis à la Direction du bureau du télégraphe à Athènes, $\mathrm{n}^{\circ} 36528 \mathrm{du}$ 6 novembre 1915.

13. AYE, 1915, Г/106, 2, Romanos à Venizélos $n^{\circ} 432$ du 10 décembre 1914 et Venizélos à Romanos, $\mathrm{n}^{\circ} 46285$ du 12 janvier 1915. AYE/Archives de l'ambassade grecque de Paris, 1916, T/4, Scouloudis à Romanos, $n^{\circ} 12607$ du 8 décembre 1915 et Romanos à Scouloudis, $n^{\circ} 191$ du 24 mars 1916.

14. AYE, 1917, Г/106, 5, Romanos à Scouloudis, $n^{\circ} 1211$ du 25 décembre 1915. AYE/Archives de l'ambassade grecque de Paris, 1916, T/4, Scouloudis à Romanos, nº 2088 du 12 mars 1916.

15. L'Humanité du 29 mai 1916, p. 1, col. 2. L'Écho de Paris du 31 mai 1916, p. 1, col. 5.

16. L'Écho de Paris du 2 juin 1916, p. 1, col. 2. Le Bulletin du jour dans Le Temps du 11 juin 1916.

17. SHD, $7 \mathrm{~N}^{\circ} 993$, Commission militaire de contrôle postal de Marseille, Rapport moral. France, établi le 15 juillet 1916.

18. BDIC, F 270 Res, C.G., le général Coquet, commandant la $15^{\mathrm{e}}$ région au directeur général des relations avec la presse à Paris, note $\mathrm{n}^{\circ} 7171$ du 27 juin 1916.

19. SHD, 5 N 342, Margerie à Roques, $n^{\circ} 5382$ et à Guillemin, $n^{\circ} 788$ du 20 juillet 1916. 
20. L'Humanité du 18 septembre 1916, p. 1, col. 2.

21. Le Bulletin du jour dans Le Temps du 28 septembre 1916.

22. Le Figaro du 28 septembre 1916, p. 1, col. 6 et p. 2, col. 1. L'éditorial dans L'Homme enchaîné du 29 septembre 1916.

23. Le Bulletin du jour dans le Temps du 28 septembre 1916.

24. Le Journal des Débats du 6 octobre 1916, p. 545.

25. Le Journal du 8 octobre 1916, p. 1, col. 2.

26. Le Petit Journal du 13 octobre 1916, p. 1, col. 1, 2. L'Homme enchaîné du 20 octobre 1916, p. 1, col. 1 et 2 .

27. SHD, 7 N 994, Contrôle postal militaire de Marseille, Grèce. Rapport économique et financier, établi le 13 octobre 1916.

28. À l'aube du $1^{\text {er }}$ décembre 1916, le gouvernement grec ayant rejeté les ultimatums successifs des Alliés concernant la cession de matériel de guerre, l'amiral Dartige du Fournet, chef commandant des forces navales alliées en Méditerranée orientale, fit débarquer une force de 3000 hommes au Phalère et au Pirée, avec pour ordre d'occuper certaines positions stratégiques à Athènes. Des chocs se sont produits autour des postes occupés par les Alliés, près du Zappeion et de la colline de Philoppapos. Deux coups de canons furent tirés d'une colline que tenaient les Grecs sur le Palais des Expositions du Zappeion où étaient cantonnés les marins français et soixante-quatre autres ont été tirés sur la ville par l'escadre. Dans la nuit, on arriva à une entente et les affrontements prirent fin. Le bilan de pertes subies des deux cotés se révéla lourd: 57 Français et 5 Britanniques furent tués, plus de 160 blessés, tandis que du côté des Grecs on comptait 45 à 50 tués et 100 à 150 blessés. Voir : SHM, SS X f 9, Roquefeuil à Lacaze, « Rapport sur les événements qui se sont déroulés dans les premiers jours de décembre 1916 ", n 533 du 9 décembre 1916. Maccas, 1916. Dartige du Fournet, 1920, 210-273. Boussenot, 1938, 1-27. Mourélos, 2007 et du même auteur, 1983, 41-45 et 1992, 111-120.

29. Le Petit Parisien du 3 décembre 1916, Le Temps du 4 décembre 1916, L'Action française du 5 décembre 1916, Le Petit Journal du 15 décembre 1916.

30. Article de Saint-Brice dans Le Journal du 4 décembre 1916, p. 1, col. 3, 4.

31. MAE, 265, Briand à Paul Cambon, s.n. Du 12 décembre 1916.

32. SHD, 7 N 996, Contrôle postal militaire de Marseille, Rapport moral et politique, établi le 15 février 1917.

33. AYE/Archives du gouvernement provisoire, 1917, A/VI, 13, A. Diomidis à Venizélos, $\mathrm{n}^{\circ} 1 \mathrm{du}$ 12 janvier 1917 et $n^{\circ} 5$ du 26 janvier 1917.

34. AYE/Archives du gouvernement provisoire, 1917, A/VI, 13, Diomidis à Venizélos, rapport du 27 mars 1917.

35. À ce moment il y avait deux légations grecques à Paris : la légation royale de la rue AugusteVacquerie, sous la direction du chargé d'affaires, Pierre Métaxas, et la légation venizéliste de l'avenue Malakoff, avec à sa tête Romanos, reconnue par le gouvernement français depuis début janvier 1917.

36. AYE, 1917, A/5/XII, P. Métaxas à Zalocostas, $n^{\circ} 703$ du 11 avril 1917. Cosmin, 1969, 351.

37. SHD, $5 \mathrm{~N}, 347$.

38. AYE, 1917, A/5/XII, Zalacostas aux légations grecques à Paris, à Londres, à Rome, à Pétrograd, à Washington, à Berne et à La Haye, $\mathrm{n}^{\circ} 2153$ du 24 avril 1917.

39. AYE, 1917, A/5/XII, P. Métaxas à Zaïmis, nº 930 du 30 avril 1917.

40. AYE, 1917, A/5/XII, P. Métaxas à Zalocostas, télégramme strictement confidentiel $n^{\circ} 829 \mathrm{du}$ 6 mai 1917.

41. SHD, $7 \mathrm{n}^{\circ} 997$, Commission militaire de contrôle postal de Marseille, rapport mensuel : État moral et politique. Grèce, établi le 15 mai 1917.

42. SHD, 7 N 997, Commission militaire de contrôle postal de Marseille, rapport mensuel : État moral et politique. Grèce, établi le 15 mai 1917. 
43. Le Bulletin du jour dans Le Temps du 14 juin 1917 et le même journal du 15 juin 1917, p. 1, col. 4.

44. Le Journal du 13 juin 1917, p. 1, col. 5. Lemonidou, 2007, 47-70, 215-249.

45. L'Action française, 13 juin 1917, p. 1, col. 4 ; La Revue bleue, nº 13, 30 juin 1917-7 juillet 1917, p. 397-398.

46. Renouvin, 1968, 14-17 et du même auteur, 1970, 321-325. Becker, 1980, 204-220 et du même auteur, 1987, 1499-1505, 1992, 98 et 1997.

47. L'éditorial de Saint-Brice dans Le Journal du 30 juin 1917. Le Petit Parisien du 26 juin 1917, p. 1, col. 2. L'Excelsior du 26 juin 1917, p. 2, col. 2.

\section{RÉSUMÉS}

L'étude de la Première Guerre mondiale montre bien que les dirigeants français furent très concernés et particulièrement impliqués dans l'affaire grecque, et qu'il en fut de même pour la presse française, qui montra pour cette conjoncture un intérêt et une curiosité extrêmes. Cette presse constitua le seul vecteur de la propagande durant la guerre et il est remarquable que l'image que l'opinion publique put percevoir de la Grèce et des relations de ce pays avec la France apparaissent homogènes à travers des journaux de tendances politiques variées. D'ailleurs, quand il s'agissait de la Grèce, ce pays sur lequel le public français ne disposait comme source d'information que de la presse, il était plutôt difficile, sinon impossible, de procéder à une lecture sélective des journaux et d'entrevoir les messages occultés de l'information officielle; c'est pourquoi la campagne que la presse française mena sur les affaires grecques porta ses fruits très tôt et sans difficulté. Notre but est d'étudier la stratégie de cette propagande par la presse française, ainsi que les efforts des dirigeants en France qui, conscients du pouvoir de la presse, utilisèrent ce moyen pour manipuler l'opinion et servir leur politique.

The study of the First World War shows clearly that the French leadership was very concerned and particularly involved in the Greek issue; at the same time, the press in France showed extremely big interest in the Greek case, too. Press was the only channel of propaganda in France during the wartime - it is remarkable that the image of Greece and of its relations with France appears have been homogeneous through newspapers of varied political stances. As the public didn't have any sources of information on Greece other than the press, it was difficult, almost impossible for them to do a selective reading of the papers and to understand the hidden messages of official information channels; that's why the campaign which the French press conducted on the Greek issue was soon and easily successful. Our aim is to study the strategy of this propaganda undertaken by the French press, as well as the efforts of the French authorities, which, being conscious of the power of press, made the most of it in order to manipulate the public opinion and support their policy.

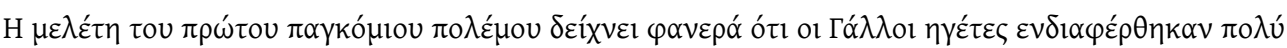

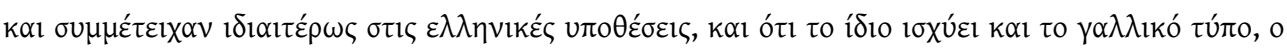

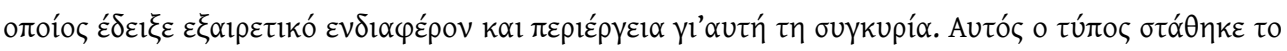

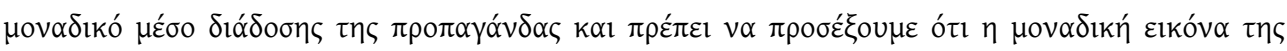

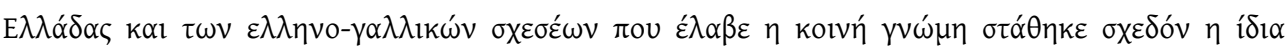

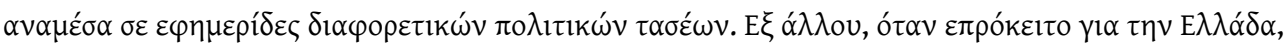

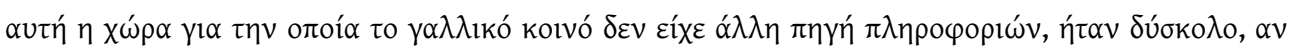




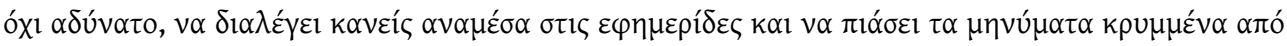

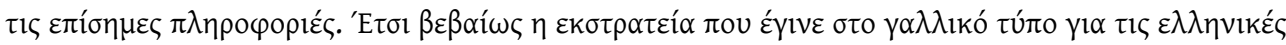

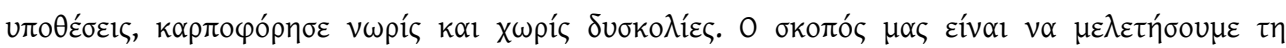

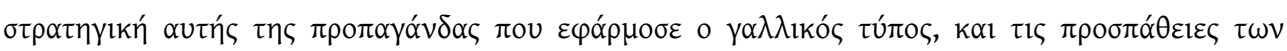

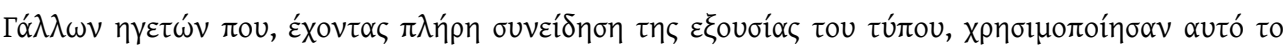

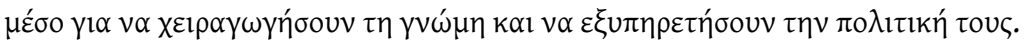

\section{INDEX}

Mots-clés : Venizélos Elefthérios (1864-1936), presse française, Constantin Ier de Grèce (1868-1923), information, opinion publique, Jonnart Charles (1857-1927), Rupel, propagande motsclesmk ФРАНЦУСКИОТ ПЕЧАТ, ИНФОРМАЦИИ, МИСЛЕЊЕ ЈАВНОСТА, ПРОПАГАНДА, ФРАНЦИЈА, ГРЦИЈА, ПРВАТА СВЕТСКА ВОЈНА, ИСТОРИЈА, ИСТОРИЈА НА ПЕЧАТОТ

motsclestr Fransız basını, Bilgi, Public görüş, Propaganda, Fransa, Yunanistan, Ilk Dünya Savaşı, Tarih, Basın Tarihi

Thèmes : Histoire, Histoire de la presse

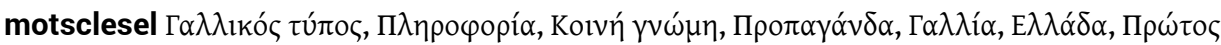

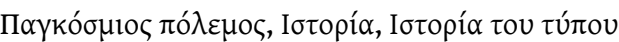

Index géographique : France, Grèce

Keywords : French press, public opinion, propaganda, France, First World War, History

Index chronologique : guerre mondiale (1914-1918) 